\title{
Numerical Solution for Solving System of Fuzzy Nonlinear Integral Equation by Using Modified Decomposition Method
}

\author{
Alan Jalal Abdulqader ${ }^{1}$ \\ ${ }^{1}$ College of education Department of Mathematics, Al-Mustansiriyah University, Iraq \\ Correspondence: Alan Jalal Abdulqader, College of education Department of Mathematics, Al-Mustansiriyah University, \\ Iraq. E-mail: alanjala1515@yahoo.com
}

\author{
Received: October 30, 2017 Accepted: November 14, $2017 \quad$ Online Published: November 29, 2017 \\ doi:10.5539/jmr.v10n1p32 \\ URL: https://doi.org/10.5539/jmr.v10n1p32
}

\begin{abstract}
In this paper, we intend to offer system of fuzzy nonlinear integral equation also numerical scheme to solve. by using the new and fast technique to solve our problem. we try to discuss some numerical aspects such as convergence and error analysis. Finally, accuracy and applicability of the proposed methods are carried out along with comparisons using some numerical examples.
\end{abstract}

Keywords: Fuzzy number; system of nonlinear Fuzzy Volterra integral equation; fuzzy integral; fuzzy Remian integral; Modified decomposition method

\section{Introduction}

In recent years, Fuzzy systems are now used to study a various of problems ranging from fuzzy metric spaces (Park, J. H., 2004), fuzzy topological spaces (Caldas, M. \& Jafari, S., 2005), to control chaotic systems (Feng, G. \& Chen, G., 2005; Jiang, W., Guo-Dong, Q. \& Bin, D. 2005), fuzzy differential equations (Abbasbandy, S. \& Allahviranloo, T., 2004; Abbasbandy, S., Nieto, J. J. \& Alavi, M., 2005)and particle physics (Naschie, M. S. El, 2004; Allahviranloo, T., Khezerloo, M., Ghanbari, M. \& Khezerloo, S., 2010). The topics of fuzzy integral equations (FIEs) which attracted growing interest for some times, particularly, in relation to fuzzy control, have been developed. The concept of integration of fuzzy functions was _firstly introduced by Dubois and Prade (1982). Alternative approaches were later suggested by Goetschel and Voxman (1986), Kaleva (1987), Matloka (1987), Seikkala (1987). Recently, some numerical methods have been introduced to solve fuzzy integral equation. For example Babolian et al (Dubois, D. \& Prade, H., 1978) used the Adomian decomposition method (ADM) to solve Fredholm fuzzy integral equations of the second kind (FFIEs-2). Allahviranloo et al (Dubois, D. \& Prade, H., 1982), nonlinear integral equations. Yalcinbas (Babolian, E., Biazar, J. \& Vahidi, A.R., 2004), as two of the most important basic polynomials which in previous works have not been covered in fuzzy equations,Modified decomposition method is proposed to solve complexity composition fuzzy kernels and complexity fuzzy nonlinear integral equation with complexly kernels, we convert a fuzzy nonlinear integral equation to a system of integral equation some numerical examples are presented to show the facts about our methods.

\section{Preliminaries}

Definition 2.1,(Jiang, W., Guo-Dong, Q. \& Bin, 2005). Fuzzy set. A set. $\widetilde{A}=\left\{\left(\mathrm{x}, \mathrm{M}_{\widetilde{A}(\mathrm{x})}\right)\right.$, $\left.\mathrm{x} \in \mathrm{X}\right\}$ is called a fuzzy set where $M_{\widetilde{A}(x)}$ is the membership function of a fuzzy set $A$ is defined by $M_{\widetilde{A}(x)}: X \rightarrow[0,1]$, the value of $M_{\widetilde{A}(x)}$ is called the membership degree $\mathrm{X}$

Definition 2.2,(Jiang, W., Guo-Dong, Q. \& Bin, 2005). Fuzzy number. A fuzzy number is a map $\widetilde{\mathbf{u}}: \mathrm{R} \rightarrow[\mathrm{a}, \mathrm{b}]$, which satisfying

(1) $\tilde{u}$ is upper semi- continuous function,

(2) $\tilde{u}(\mathrm{x})=0$ outside some interval $[\mathrm{a}, \mathrm{d}]$

(3) There are real numbers b,c such a $\leq$ b $\leq$ c $\leq$ d

i) $\quad \tilde{u}(\mathrm{x})$ is a monotonic increasing function on $[\mathrm{a}, \mathrm{b}]$

ii) $\quad \tilde{u}(\mathrm{x})$ is a monotonic decreasing function on $[\mathrm{c}, \mathrm{d}]$

iii) $\tilde{u}(\mathrm{x})=1$ for all $\mathrm{x} \in[\mathrm{b}, \mathrm{c}]$

The set of all fuzzy numbers (as given by Definition 1.2 ) is denoted by $\mathrm{E}^{1}$ and is a convex cone. An alternative definition 
for parameter from of a fuzzy number is given by Kaleva [33].

Definition 2.3, (Abbasbandy, S., Nieto, J. J. \& Alavi, M., 2005). fuzzy interval. Let u be a fuzzy set on R. u is called a fuzzy interval if satisfied

$\mathrm{u}$ is normal: there exsist $\mathrm{x}_{0} \in \mathrm{R}$ S.T $\mathrm{u}\left(\mathrm{x}_{0}\right)=1$

$\mathrm{u}$ is convex: for all $\mathrm{x}, \mathrm{t} \in \mathrm{R}, 0 \leq \lambda \leq 1$ it holds that $\mathrm{u}(\lambda \mathrm{x}+(1-\lambda) \mathrm{y} \geq \min \{\mathrm{u}(\mathrm{x}), \mathrm{u}(\mathrm{y})\}$

$\mathrm{u}$ is upper semi- continuous

$[\mathrm{u}]^{0}=\mathrm{cl}\{\mathrm{x} \in \mathrm{R}: \mathrm{u}(\mathrm{x})>0\}$ is a compact subset of $\mathrm{R}$

Definition 2.4, (Abbasbandy, S., Nieto, J. J. \& Alavi, M., 2005). $\alpha$ - level.

let $\mathrm{E}$ be the set of all fuzzy number on $\mathrm{R}$, the $\alpha$ - level set of a fuzzy number $\mathrm{u} \in \mathrm{E}, 0 \leq \alpha \leq 1$, denoted by $[u]^{\alpha}$ is mapping between close interval 0,1 to power set of $R$ is defined as

$$
[\mathrm{u}]^{\alpha}=\left\{\begin{array}{cl}
{[\mathrm{a}(\alpha), \mathrm{b}(\alpha)],} & \alpha \in(0.1] \\
\mathrm{cl}(\operatorname{supp}), & \alpha=0
\end{array}\right.
$$

The $\alpha$ - level set of a fuzzy number is closed and bounded interval $[\underline{u}(\alpha), \bar{u}(\alpha)]$, where $\underline{u}(\alpha)$ denotes the left-hand endpoint of $[\mathrm{u}]^{\alpha}$ and $\overline{\mathrm{u}}(\alpha)$ denotes the right - hand endpoint of $[\mathrm{u}]^{\alpha}$ since each $\mathrm{y} \in \mathrm{R}$ can be regarded as a fuzzy number $\tilde{y}$ defined by $\tilde{y}(t)=\left\{\begin{array}{l}1 \text { if } t=y \\ 0 \text { if } t \neq y\end{array}\right.$

Definition 2.5, (Naschie, M. S. El, 2004). A fuzzy number $\breve{u}$ in parametric form is a pair $(\underline{u}, \bar{u})$ of function $\underline{u}(\alpha), \bar{u}(\alpha)$, $0 \leq \alpha \leq 1$, which satisfies the following requiremenst:

i) $\underline{\mathrm{u}}(\alpha)$ is a bounded left continuous non- decreasing function over $[0,1]$

ii) $\overline{\mathrm{u}}(\alpha)$ is a bounded left continuous non- increasing function over $[0,1]$

iii) $\underline{\mathrm{u}}(\alpha) \leq \overline{\mathrm{u}}(\alpha), 0 \leq \alpha \leq 1$

Definition 2.6, (Goetschel, R. \& Voxman, W., 1986).For arbitrary fuzzy $u=(\underline{u}(\alpha), \bar{u}(\alpha)), v=(\underline{v}(\alpha), \bar{v}(\alpha)), 0 \leq \alpha \leq$ 1 and scalar $\mathrm{k}$, we define addition, subtraction, scalar product by $\mathrm{k}$ and multiplication are respectively as following:

$1-$ addition: $(\underline{\mathrm{u}+\mathrm{v})}(\alpha)=(\underline{\mathrm{u}}(\alpha)+\underline{\mathrm{v}}(\alpha)), \quad(\overline{\mathrm{u}+\mathrm{v})}(\alpha)=(\overline{\mathrm{u}}(\alpha)+\overline{\mathrm{v}}(\alpha))$,

2 - subtraction: $(\bar{u}-v)(\alpha)=(\underline{u}(\alpha)-\underline{v}(\alpha)), \quad(\overline{u-v)}(\alpha)=(\bar{u}(\alpha)-\bar{v}(\alpha))$,

3 - scalar product :

$$
k \breve{u}=\left\{\begin{array}{cc}
(\underline{k} \underline{u}(\alpha), k \bar{u}(\alpha)), & k \geq 0 \\
(k \underline{u}(\alpha), k \bar{u}(\alpha)), & k<0
\end{array}\right.
$$

4- multiplication:

$$
\tilde{\mathrm{u}} \cdot \tilde{\mathrm{v}}=\left\{\begin{array}{l}
\underline{\mathrm{uv}}(\alpha)=\max \{\underline{\mathrm{u}}(\alpha) \underline{\mathrm{v}}(\alpha), \underline{\mathrm{u}}(\alpha) \overline{\mathrm{v}}(\alpha), \overline{\mathrm{u}}(\alpha) \underline{\mathrm{v}}(\alpha), \overline{\mathrm{u}}(\alpha) \overline{\mathrm{v}}(\alpha)\} \\
\overline{\mathrm{uv}}(\alpha)=\min \{\underline{\mathrm{u}}(\alpha) \underline{\mathrm{v}}(\alpha), \underline{\mathrm{u}}(\alpha) \overline{\mathrm{v}}(\alpha), \overline{\mathrm{u}}(\alpha) \underline{\mathrm{v}}(\alpha), \overline{\mathrm{u}}(\alpha) \overline{\mathrm{v}}(\alpha)\}
\end{array}\right.
$$

Definition 2.7,( Dubois, D. \& Prade, H., 1982). For arbitrary Fuzzy numbers $\tilde{u}, \tilde{v} \in E^{1}$, we use the distance

$$
\mathrm{D}(\tilde{u}, \tilde{\mathrm{v}})=\max \left\{\sup _{\alpha \in[0,1]}|\underline{\mathrm{u}}(\alpha)-\underline{\mathrm{v}}(\alpha)|, \sup _{\alpha \in[0,1]}|\overline{\mathrm{u}}(\alpha)-\overline{\mathrm{v}}(\alpha)|\right\}
$$

And it is show [43] that $\left(\mathrm{E}^{1}, \mathrm{D}\right)$ is a complete metric space .

Definition 2.8, (Dubois, D., Prade, 1986). let $\tilde{\mathrm{f}}(\mathrm{x})$ be a close and bounded fuzzy value function on $[\mathrm{a}, \mathrm{b}]$. Suppose the $\tilde{\mathrm{f}}_{\alpha}^{\mathrm{L}}(\mathrm{x})$ and $\tilde{\mathrm{f}}_{\alpha}^{\mathrm{R}}(\mathrm{x})$ are a Riemann - integral on [a,b] for all $\alpha \in[0,1],[42]$.

Let

$$
\mathrm{A}_{\alpha}=\left[\int_{\mathrm{a}}^{\mathrm{b}} \tilde{\mathrm{f}}_{\alpha}^{\mathrm{L}}(\mathrm{x}) \mathrm{dx}, \int_{\mathrm{a}}^{\mathrm{b}} \tilde{\mathrm{f}}_{\alpha}^{\mathrm{R}}(\mathrm{x}) \mathrm{dx}\right]
$$

Then we say that $\tilde{\mathrm{f}}(\mathrm{x})$ is fuzzy -Riemann - integral of $[a, b]$, and the membership function of $\int_{a}^{b} \tilde{f}(x) d x$ is defined by 
$\mathrm{M}_{\int_{\mathrm{a}}^{b} \tilde{\mathrm{f}}(\mathrm{x}) \mathrm{dx}}(\alpha)=\sup _{0 \leq \alpha \leq 1} \alpha \cdot 1_{\mathrm{A}_{\alpha}}(r) \quad$ for $r \in A_{0}$

Definition 2.9, (Dubois, D., Prade, 1986). The integral of a fuzzy function was define in [16] by using the Riemann integral concept. Let $\mathrm{f}:[\mathrm{a}, \mathrm{b}] \rightarrow \mathrm{E}^{1}$. For Fuzzy function, for each partition $\mathrm{p}=\left\{\mathrm{t}_{0}, \ldots, \mathrm{t}_{\mathrm{n}}\right\}$ of $[\mathrm{a}, \mathrm{b}]$ and for arbitrary $\xi_{\mathrm{i}} \in\left[\mathrm{t}_{\mathrm{i}-1}, \mathrm{t}_{\mathrm{i}}\right], 1 \leq \mathrm{i} \leq \mathrm{n}$, suppose

$$
\begin{gathered}
\mathrm{R}_{\mathrm{P}}=\sum_{\mathrm{i}=1}^{\mathrm{n}} \mathrm{f}\left(\xi_{\mathrm{i}}\right)\left(\mathrm{t}_{\mathrm{i}}-\mathrm{t}_{\mathrm{i}-1}\right) \\
\Delta:=\max \left\{\left|\mathrm{t}_{\mathrm{i}}-\mathrm{t}_{\mathrm{i}-1}\right|, 1 \leq \mathrm{i} \leq \mathrm{n}\right\} .
\end{gathered}
$$

The define integral of $f(t)$ over $[a, b]$ is

$$
\int_{a}^{b} f(t) d t=\lim _{\Delta \rightarrow 0} R_{P}
$$

If the fuzzy function $\mathrm{f}(\mathrm{t})$. is continuous in metric $\mathrm{D}$,its definite the integral exists and also

$$
\begin{aligned}
& \left(\int_{a}^{b} f(t ; \alpha) d t\right)=\int_{a}^{b} f(t ; \alpha) d t, \\
& \left(\int_{a}^{\bar{b}} \overline{\overline{f(t ; \alpha)}} d t\right)=\int_{a}^{\bar{b}} \bar{f}(t ; \alpha) d t
\end{aligned}
$$

It should be noted that the fuzzy integral can be also defined using the Lebesgue - type approach. However, if $f(t)$ is continuous, both approaches yield the same value.More details about the properties of the fuzzy integral.

Definition 2.10, (Tanaka, Y., Mizuno, Y. \& Kado, T., 2005). Let $F: I \rightarrow E^{n}$ the integral of $F$ over, denoted by

$\int_{I} F(x) d x$ or $\int_{a}^{b} F(x) d x$, is defined levelwise by

$\left[\int_{I} F(x) d x\right]^{\alpha}=\int_{I} F(x)_{\alpha} d x=\left\{\int_{I} f(x) d x \mid f: I \rightarrow R^{n}\right.$ is a masuarsble function for $F(x)_{\alpha}$ for all $0 \leq \alpha \leq 1$

Proposition 2.1 (Tanaka, Y., Mizuno, Y. \& Kado, T., 2005). A function $F, G: I \rightarrow E^{n}$ be integrable and $\varphi \in R . \in$ then

$1-\int(F+G)=\int F+\int G$

$2-\int \varphi F=\varphi \int F$

3-D $(F, G)$ IS integrable

$4-D\left(\int F, \int G\right) \leq \int D(F, G)$

Theorem 2.1.(Naschie, M. S. El, 2004) For any $p, q, r, s \in E^{n}$ and $\varphi \in R$, then the following hold

$\mathrm{i}-\left(E^{n}, D\right)$ is a complete metric space

ii- $D(\varphi p, \varphi q)=|\varphi| D(p, q)$

iii- $D(p+r, q+s)=D(p, q)$

iv- $D(p+q, r+s) \leq D(p, r)+D(q, s)$

\section{Fuzzy System of Nonlinear Volterra Integral Equation}

The fuzzy system of nonlinear integral equation with integral kernel which is discussed in this work is the fuzzy system of nonlinear Volterra integral equation as follows:

$$
\widetilde{\mathrm{u}_{1}}(\mathrm{x})=\tilde{\mathrm{f}}_{1}(\mathrm{x})+\sum_{j=1}^{m} \lambda_{1 j} \int_{\mathrm{a}}^{\mathrm{x}} \mathrm{k}_{1 j}\left(\mathrm{x}, \mathrm{t}, \widetilde{\mathrm{G}}_{1 j}\left(\mathrm{t}, \tilde{\mathrm{F}}\left(\mathrm{t}, \tilde{u}_{j}(\mathrm{t})\right)\right)\right) \mathrm{dt}
$$

$\vdots$

$$
\widetilde{\mathrm{u}}_{\mathrm{i}}(\mathrm{x})=\tilde{\mathrm{f}}_{\mathrm{i}}(\mathrm{x})+\sum_{j=1}^{m} \lambda_{\mathrm{i} j} \int_{\mathrm{a}}^{\mathrm{x}} \mathrm{k}_{\mathrm{i} j}\left(\mathrm{x}, \mathrm{t}, \widetilde{\mathrm{G}}_{\mathrm{ij}}\left(\mathrm{t}, \tilde{\mathrm{F}}\left(\mathrm{t}, \tilde{\mathrm{u}}_{j}(\mathrm{t})\right)\right)\right) \mathrm{dt}
$$

$$
\widetilde{\mathrm{u}_{m}}(\mathrm{x})=\tilde{\mathrm{f}}_{m}(\mathrm{x})+\sum_{j=1}^{m} \lambda_{m j} \int_{\mathrm{a}}^{\mathrm{x}} \mathrm{k}_{m j}\left(\mathrm{x}, \mathrm{t}, \widetilde{\mathrm{G}}_{\mathrm{mj}}\left(\mathrm{t}, \tilde{\mathrm{F}}\left(\mathrm{t}, \tilde{u}_{j}(\mathrm{t})\right)\right)\right) \mathrm{dt}
$$


where $\lambda_{i j}>0($ for $\mathrm{i}, \mathrm{j}=1, \ldots, \mathrm{m}), \tilde{\mathrm{f}}_{i}(\mathrm{x})$ is a fuzzy function of $\mathrm{x}$ such that $\mathrm{x}, \mathrm{t} \in[\mathrm{a}, \mathrm{b}]$, also $\mathrm{k}_{i j}\left(\mathrm{x}, \mathrm{t}, \widetilde{\mathrm{G}}_{\mathrm{ij}}\left(\mathrm{t}, \tilde{\mathrm{F}}\left(\mathrm{t}, \tilde{u}_{j}(\mathrm{t})\right)\right)\right)$ :

$\mathrm{I} \times \mathrm{I} \times \mathrm{E}^{1} \rightarrow \mathrm{E}^{1}$ is analytic functions, $\tilde{\mathrm{F}}\left(\mathrm{t}, \tilde{u}_{j}(\mathrm{t})\right): \mathrm{I} \times \mathrm{E}^{1} \rightarrow \mathrm{E}^{1}$ is fuzzy continuous functions, consider the pairs $\tilde{\mathrm{f}}_{i}(\mathrm{x})=\left(\underline{\mathrm{f}}_{i}(\mathrm{x}, \alpha), \overline{\mathrm{f}}_{i}(\mathrm{x}, \alpha)\right)$ and $\tilde{\mathrm{u}}_{i}(\mathrm{t})=\left(\underline{\mathrm{u}}_{i}(\mathrm{x}, \alpha), \overline{\mathrm{u}}_{i}(\mathrm{x}, \alpha)\right), 0 \leq \alpha \leq 1$ and $\mathrm{x} \in \mathrm{I}=[\mathrm{a}, \mathrm{b}]$, is solution will be determined. Then, the parametric form of Eq. (2) is given fuzzy integral equations system as follows.

$$
\begin{aligned}
\underline{\mathrm{u}}_{m}(\mathrm{x}, \alpha)=\underline{\mathrm{f}}_{m}(\mathrm{x}, \alpha)+\sum_{j=1}^{m} \lambda_{m j} & \frac{\int_{\mathrm{a}}^{\mathrm{x}} \mathrm{k}_{m j}\left(\mathrm{x}, \mathrm{t}, \mathrm{G}_{\mathrm{mj}}\left(\mathrm{t}, \mathrm{F}\left(\mathrm{t}, \mathrm{u}_{j m}(\mathrm{t}, \alpha)\right)\right) \mathrm{dt}\right.}{\overline{\mathrm{u}}_{m}(\mathrm{x}, \alpha)}=\overline{\mathrm{f}}_{m}(\mathrm{x}, \alpha)+\sum_{j={ }^{\prime}}^{m} \lambda_{m j} \overline{\int_{\mathrm{a}}^{\mathrm{x}} \mathrm{k}_{m j}\left(\mathrm{x}, \mathrm{t}, \mathrm{G}_{\mathrm{mj}}\left(\mathrm{t}, \mathrm{F}\left(\mathrm{t}, \mathrm{u}_{j m}(\mathrm{t}, \alpha)\right)\right)\right) \mathrm{dt}}
\end{aligned}
$$

We can see that Eq. (1) convert to a system of nonlinear Volterra integral equations in crisp case in Eq (3). We have :

$$
\begin{aligned}
& \underline{\mathrm{u}}_{m}(\mathrm{x}, \alpha)=\underline{\mathrm{f}}_{m}(\mathrm{x}, \alpha)+\sum_{j=1}^{m} \lambda_{m j} \int_{\mathrm{a}}^{\mathrm{c}} \mathrm{k}_{m j}\left(\mathrm{x}, \mathrm{t}, \underline{\mathrm{G}_{\mathrm{mj}}\left(\mathrm{t}, \mathrm{F}\left(\mathrm{t}, \mathrm{u}_{j m}(\mathrm{t}, \alpha)\right)\right)} \mathrm{dt}+\sum_{j=1}^{m} \lambda_{m j} \int_{\mathrm{c}}^{\mathrm{x}} \mathrm{k}_{m j}\left(\mathrm{x}, \mathrm{t}, \overline{\mathrm{G}_{\mathrm{mj}}\left(\mathrm{t}, \mathrm{F}\left(\mathrm{t}, \mathrm{u}_{j m}(\mathrm{t}, \alpha)\right)\right) d t}\right.\right. \\
& \overline{\mathrm{u}}_{m}(\mathrm{x}, \alpha)= \\
& \overline{\mathrm{f}}_{m}(\mathrm{x}, \alpha)+\sum_{j=1}^{m} \lambda_{m j} \int_{\mathrm{a}}^{\mathrm{c}} \mathrm{k}_{m j}\left(\mathrm{x}, \mathrm{t}, \overline{\mathrm{G}_{\mathrm{mj}}\left(\mathrm{t}, \mathrm{F}\left(\mathrm{t}, \mathrm{u}_{j m}(\mathrm{t}, \alpha)\right)\right)} \mathrm{dt}+\sum_{j=1}^{m} \lambda_{m j} \int_{\mathrm{c}}^{\mathrm{x}} \mathrm{k}_{m j}\left(\mathrm{x}, \mathrm{t}, \underline{\mathrm{G}_{\mathrm{mj}}\left(\mathrm{t}, \mathrm{F}\left(\mathrm{t}, \mathrm{u}_{j m}(\mathrm{t}, \alpha)\right)\right) d t}\right.\right.
\end{aligned}
$$

where $0 \leq \mathrm{t} \leq \mathrm{c}, \mathrm{c} \leq \mathrm{t} \leq \mathrm{x}, 0 \leq \alpha \leq 1$. Now we will find the parameter for the $\mathrm{Eq}(4)$, as follows

$$
\begin{aligned}
& \mathrm{k}_{m j}\left(\mathrm{x}, \mathrm{t}, \mathrm{G}_{\mathrm{mj}}\left(\mathrm{t}, \mathrm{F}\left(\mathrm{t}, \mathrm{u}_{j m}(\mathrm{t}, \alpha)\right)\right)\right)
\end{aligned}
$$

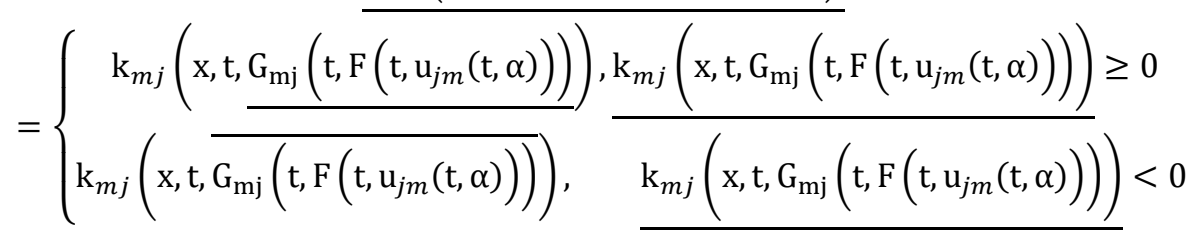

$$
\begin{aligned}
& \underline{G_{m j}\left(t, F\left(t, u_{j m}(t, \alpha)\right)\right)}=\left\{\begin{array}{l}
\frac{G_{m j}\left(t, \underline{F}\left(t, \underline{u}_{j m}(t, \alpha)\right)\right),}{G_{m j}\left(t, F\left(t, u_{j m}(t, \alpha)\right)\right)} \geq 0 \\
\underline{G}_{m j}\left(t, \bar{F}\left(t, \bar{u}_{j m}(t, \alpha)\right)\right), \underline{G_{m j}\left(t, F\left(t, u_{j m}(t, \alpha)\right)\right)}<0
\end{array}\right. \\
& \overline{G_{m j}\left(t, F\left(t, u_{j m}(t, \alpha)\right)\right)}=\left\{\begin{array}{l}
\bar{G}_{m j}\left(t, \bar{F}\left(t, \bar{u}_{j m}(t, \alpha)\right)\right), \overline{G_{m j}\left(t, F\left(t, u_{j m}(t, \alpha)\right)\right)} \geq 0 \\
\bar{G}_{m j}\left(t, \underline{F}\left(t, \underline{u}_{j m}(t, \alpha)\right)\right), \overline{G_{m j}\left(t, F\left(t, u_{j m}(t, \alpha)\right)\right)}<0
\end{array}\right. \\
& \overline{\mathrm{k}_{m j}\left(\mathrm{x}, \mathrm{t}, \mathrm{G}_{\mathrm{mj}}\left(\mathrm{t}, \mathrm{F}\left(\mathrm{t}, \mathrm{u}_{j m}(\mathrm{t}, \alpha)\right)\right)\right)}=\left\{\begin{array}{l}
\left.\mathrm{k}_{m j}\left(\mathrm{x}, \mathrm{t}, \overline{\mathrm{G}_{\mathrm{mj}}\left(\mathrm{t}, \mathrm{F}\left(\mathrm{t}, \mathrm{u}_{j m}(\mathrm{t}, \alpha)\right)\right)}\right) \overline{, \mathrm{k}_{m j}\left(\mathrm{x}, \mathrm{t}, \mathrm{G}_{\mathrm{mj}}\left(\mathrm{t}, \mathrm{F}\left(\mathrm{t}, \mathrm{u}_{j m}(\mathrm{t}, \alpha)\right)\right)\right.}\right) \\
\mathrm{k}_{m j}\left(\mathrm{x}, \mathrm{t}, \overline{\mathrm{G}_{\mathrm{mj}}\left(\mathrm{t}, \mathrm{F}\left(\mathrm{t}, \mathrm{u}_{j m}(\mathrm{t}, \alpha)\right)\right)}\right) \overline{, \mathrm{k}_{m j}\left(\mathrm{x}, \mathrm{t}, \mathrm{G}_{\mathrm{mj}}\left(\mathrm{t}, \mathrm{F}\left(\mathrm{t}, \mathrm{u}_{j m}(\mathrm{t}, \alpha)\right)\right)\right.}<0
\end{array}\right. \\
& \overline{G_{m j}\left(t, F\left(t, u_{j n-1}(t, \alpha)\right)\right)}= \begin{cases}\bar{G}_{m j}\left(t, \bar{F}\left(t, \bar{u}_{j m}(t, \alpha)\right)\right), & \overline{G_{j m}\left(t, F\left(t, u_{j m}(t, \alpha)\right)\right)} \geq 0 \\
\bar{G}_{m j}\left(t, \underline{F}\left(t, \underline{u}_{j m}(t, \alpha)\right)\right), \overline{G_{m j}\left(t, F\left(t, u_{j m}(t, \alpha)\right)\right)}<0\end{cases}
\end{aligned}
$$




$$
\underline{\mathrm{G}_{\mathrm{mj}}\left(\mathrm{t}, \mathrm{F}\left(\mathrm{t}, \mathrm{u}_{j m}(\mathrm{t}, \alpha)\right)\right)}=\left\{\begin{array}{l}
\underline{\mathrm{G}}_{\mathrm{mj}}\left(\mathrm{t}, \underline{\mathrm{F}}\left(\mathrm{t}, \underline{\mathrm{u}}_{j m}(\mathrm{t}, \alpha)\right)\right), \underline{\mathrm{G}_{\mathrm{mj}}\left(\mathrm{t}, \mathrm{F}\left(\mathrm{t}, \mathrm{u}_{j m}(\mathrm{t}, \alpha)\right)\right) \geq 0} \\
\underline{G}_{\mathrm{mj}}\left(\mathrm{t}, \overline{\mathrm{F}}\left(\mathrm{t}, \overline{\mathrm{u}}_{j m}(\mathrm{t}, \alpha)\right)\right), \underline{\mathrm{G}_{\mathrm{mj}}\left(\mathrm{t}, \mathrm{F}\left(\mathrm{t}, \mathrm{u}_{j m}(\mathrm{t}, \alpha)\right)\right)}<0
\end{array}\right.
$$

Where $\mathrm{j}=1, \ldots, \mathrm{m}$

than

$$
\begin{gathered}
\underline{\mathrm{u}}(\mathrm{x}, \alpha)=\underline{\mathrm{f}}_{m}(\mathrm{x}, \alpha)+\sum_{j=1}^{m}\left[\int _ { \mathrm { a } } ^ { \mathrm { d } } \mathrm { k } _ { m j } \left(\mathrm{x}, \mathrm{t}, \underline{\mathrm{G}_{\mathrm{mj}}}\left(\mathrm{t}, \underline{\mathrm{F}}\left(\mathrm{t}, \underline{\mathrm{u}}_{j m}(\mathrm{t}, \alpha)\right)\right) \mathrm{dt}+\int_{\mathrm{d}}^{\mathrm{c}} \mathrm{k}_{m j}\left(\mathrm{x}, \mathrm{t}, \overline{\mathrm{G}}_{\mathrm{j}}\left(\mathrm{t}, \overline{\mathrm{F}}\left(\mathrm{t}, \overline{\mathrm{u}}_{j m}(\mathrm{t}, \alpha)\right)\right) \mathrm{dt}+\right.\right.\right. \\
\int_{\mathrm{c}}^{\mathrm{e}} \mathrm{k}_{m j}\left(\mathrm{x}, \mathrm{t}, \underline{\mathrm{G}}_{\mathrm{mj}}\left(\mathrm{t}, \overline{\mathrm{F}}\left(\mathrm{t}, \overline{\mathrm{u}}_{j m}(\mathrm{t}, \alpha)\right)\right) \mathrm{dt}+\int_{\mathrm{e}}^{\mathrm{x}} \mathrm{k}_{m j}\left(\mathrm{x}, \mathrm{t}, \overline{\mathrm{G}}_{\mathrm{mj}}\left(\mathrm{t}, \underline{\mathrm{F}}\left(\mathrm{t}, \underline{\mathrm{u}}_{j m}(\mathrm{t}, \alpha)\right)\right) \mathrm{dt}\right]\right. \\
\overline{\mathrm{u}_{m}}(\mathrm{x}, \alpha)=\overline{\mathrm{f}}_{m}(\mathrm{x}, \alpha)+\sum_{j=1}^{m}\left[\int _ { \mathrm { a } } ^ { \mathrm { d } } \mathrm { k } _ { m j } \left(\mathrm{x}, \mathrm{t}, \overline{\mathrm{G}}_{\mathrm{mj}}\left(\mathrm{t}, \overline{\mathrm{F}}\left(\mathrm{t}, \overline{\mathrm{u}}_{j m}(\mathrm{t}, \alpha)\right)\right) d t+\int_{\mathrm{d}}^{\mathrm{c}} \mathrm{k}_{m j}\left(\mathrm{x}, \mathrm{t}, \mathrm{k}\left(\mathrm{x}, \mathrm{t}, \underline{\mathrm{G}_{\mathrm{mj}}}\left(\mathrm{t}, \underline{\mathrm{F}}\left(\mathrm{t}, \underline{\mathrm{u}}_{j m}(\mathrm{t}, \alpha)\right)\right) d t+\right.\right.\right.\right. \\
\int_{\mathrm{c}}^{\mathrm{e}} \mathrm{k}_{m j}\left(\mathrm{x}, \mathrm{t}, \overline{\mathrm{G}}_{\mathrm{mj}}\left(\mathrm{t}, \underline{\mathrm{F}}\left(\mathrm{t}, \underline{\mathrm{u}}_{j m}(\mathrm{t}, \alpha)\right)\right) \mathrm{dt}+\int_{\mathrm{e}}^{\mathrm{x}} \mathrm{k}_{m j}\left(\mathrm{x}, \mathrm{t}, \underline{\mathrm{G}}_{\mathrm{mj}}\left(\mathrm{t}, \overline{\mathrm{F}}\left(\mathrm{t}, \overline{\mathrm{u}}_{j m}(\mathrm{t}, \alpha)\right)\right) \mathrm{dt}\right]\right.
\end{gathered}
$$

where $0 \leq \mathrm{t} \leq \mathrm{d}, \mathrm{d} \leq \mathrm{t} \leq \mathrm{c}, \mathrm{c} \leq \mathrm{t} \leq \mathrm{e}, \mathrm{e} \leq \mathrm{t} \leq \mathrm{x}, 0 \leq \alpha \leq 1$

This is the condition for the fuzzy nonlinear integral system. If $i=n$ that mean we will reply equation (3) and (4) for all time $\mathrm{n}$, for example if $\mathrm{i}=2$ is mean we have two equations in fuzzy type and the parametric from equation (6) from $\widetilde{f_{m}}(x)=$ $\left(\underline{\mathrm{f}}_{m}(\mathrm{x}, \alpha), \overline{\mathrm{f}}_{m}(\mathrm{x}, \alpha)\right)$ and $\widetilde{u_{m}}(x)=\left(\underline{\mathrm{u}_{m}}(\mathrm{x}, \alpha), \overline{\mathrm{u}_{m}}(\mathrm{x}, \alpha)\right)$ respectively .

The equation (1) is convert to the system of nonlinear volterra integral equations in crisp case. Now we will explain modified decomposition method to solve our system and find the approximate solution for $\tilde{u}(\mathrm{x}) a \leq x \leq b$.

\section{Modified Decomposition Method}

The Adomian asserts that the decomposition method provides an efficient and computationally convenient method for generating approximate series solution to a widely class of equations. The method is applied as follows:

Standard Adomian method define the solution $\underline{u}(x, \alpha), \bar{u}(x, \alpha)$ the series

$$
\left\{\begin{array}{c}
\underline{u}(x, \alpha)=\sum_{i=0}^{\infty} \underline{u}_{i}(x, \alpha) \\
\bar{u}(x, \alpha)=\sum_{i=0}^{\infty} \bar{u}_{i}(x, \alpha) \\
0 \leq \alpha \leq 1
\end{array}\right.
$$

Substitution the series decomposition (10) into both side of $\operatorname{Eq}(7)$ and assuming that the function $\underline{f}_{i}, \bar{f}_{i}$ can be expressed as the sum of two part $\underline{f}_{i 0}, \bar{f}_{i 0}, \underline{f}_{i 1}, \bar{f}_{i 1}$, therefore used

$$
\begin{aligned}
\underline{f_{i}} & =\underline{f}_{i 0}+\underline{f}_{i 1}, \mathrm{i}=1,2,3, \ldots, \mathrm{m} \\
\bar{f}_{i} & =\bar{f}_{i 0}+\bar{f}_{i 1}
\end{aligned}
$$

In view of the assumption, we propose a slight variation is that only the part $f_{i 0}, \bar{f}_{i 0}$ is assigned to the zeroth component $\underline{u}_{i 0}, \bar{u}_{i 0}$ and the remaining part $\underline{f}_{i 1}, \bar{f}_{i 1}$ are combined with the others terms ( integral part ) to define $\underline{u}_{1 i r}, \bar{u}_{1 i r}$, based on the suggestion formulate the following modified decomposition methods.rewrite equation (5). We get $\sum_{i=0}^{\infty} \underline{u_{i m}}(\mathrm{x}, \alpha)=$

$$
\begin{gathered}
\underline{f}_{i 0}(\mathrm{x}, \alpha)+\underline{f}_{\underline{\mathrm{i}} 1}(\mathrm{x}, \alpha)+ \\
\sum_{j=1}^{m}\left[\int _ { \mathrm { a } } ^ { \mathrm { d } } \mathrm { k } _ { m j } \left(\mathrm{x}, \mathrm{t}, \underline{\mathrm{G}_{\mathrm{mj}}}\left(\mathrm{t}, \underline{\mathrm{F}}\left(\mathrm{t},\left[R \sum_{i=0}^{\infty} \underline{\mathrm{u}}_{j m-1 i}(\mathrm{t}, \alpha)+N \sum_{i=0}^{\infty} \underline{\mathrm{u}}_{j m-1 i}(\mathrm{t}, \alpha)\right)\right]\right) \mathrm{dt}+\right.\right.
\end{gathered}
$$




$$
\begin{gathered}
\int_{\mathrm{d}}^{\mathrm{c}} \mathrm{k}_{m j}\left(\mathrm{x}, \mathrm{t}, \overline{\mathrm{G}}_{\mathrm{mj}}\left(\mathrm{t}, \overline{\mathrm{F}}\left(\mathrm{t},\left[R \sum_{i=0}^{\infty} \overline{\mathrm{u}}_{j m-1 i}(\mathrm{t}, \alpha)+\right.\right.\right.\right. \\
\left.\left.\left.N \sum_{i=0}^{\infty} \overline{\mathrm{u}}_{(j m-1) i}(\mathrm{t}, \alpha)\right]\right)\right) \mathrm{dt}+\int_{\mathrm{c}}^{\mathrm{e}} \mathrm{k}_{m j}\left(\mathrm{x}, \mathrm{t}, \underline{\mathrm{G}}_{\mathrm{mj}}\left(\mathrm{t}, \overline{\mathrm{F}}\left(\mathrm{t},\left[R \sum_{i=0}^{\infty} \overline{\mathrm{u}}_{(j m-1) i}(\mathrm{t}, \alpha)+N \sum_{i=0}^{\infty} \overline{\mathrm{u}}_{(j m-1) i}(\mathrm{t}, \alpha)\right]\right)\right) \mathrm{dt}+\right. \\
\int_{\mathrm{e}}^{\mathrm{x}} \mathrm{k}_{m j}\left(\mathrm{x}, \mathrm{t}, \overline{\mathrm{G}}_{\mathrm{mj}}\left(\mathrm{t}, \underline{\mathrm{F}}\left(\mathrm{t},\left[R \sum_{i=0}^{\infty} \underline{\mathrm{u}}_{(j m-1) i}(\mathrm{t}, \alpha)+N \sum_{i=0}^{\infty} \underline{\mathrm{u}}_{(j m-1) i}(\mathrm{t}, \alpha)\right]\right)\right) \mathrm{dt}\right] \\
\sum_{i=0}^{\infty} \overline{\mathrm{u}} \overline{\mathrm{u}_{m}}(\mathrm{x}, \alpha)= \\
\bar{f}_{i 0}(\mathrm{x}, \alpha)+\bar{f}_{i 1}(\mathrm{x}, \alpha)+\sum_{j=1}^{m}\left[\int _ { \mathrm { a } } ^ { \mathrm { d } } \mathrm { k } _ { m j } \left(\mathrm{x}, \mathrm{t}, \overline{\mathrm{G}}_{\mathrm{mj}}\left(\mathrm{t}, \overline{\mathrm{F}}\left(\mathrm{t},\left[R \sum_{i=0}^{\infty} \overline{\mathrm{u}}_{(j m-1) i}(\mathrm{t}, \alpha)+N \sum_{i=0}^{\infty} \overline{\mathrm{u}}_{(j m-1) i}(\mathrm{t}, \alpha)\right]\right)\right) d t+\right.\right. \\
\int_{\mathrm{c}}^{\mathrm{e}} \mathrm{k}_{m j}\left(\mathrm{x}, \mathrm{t}, \overline{\mathrm{G}}_{\mathrm{mj}}\left(\mathrm{t}, \underline{\mathrm{F}}\left(\mathrm{t},\left[R \sum_{i=0}^{\infty} \underline{\mathrm{u}}_{(j m-1) i}(\mathrm{t}, \alpha)+\right.\right.\right.\right. \\
\left.\left.\left.\left.N \sum_{r=0}^{\infty} \underline{\mathrm{u}}_{(j m-1) i}(\mathrm{t}, \alpha)\right]\right)\right) \mathrm{dt}+\int_{\mathrm{e}}^{\mathrm{x}} \mathrm{k}_{m j}\left(\mathrm{t}, \underline{\mathrm{F}}\left(\mathrm{t},\left[R \sum_{i=0}^{\infty} \underline{\mathrm{u}}_{(j m-1) i}(\mathrm{t}, \alpha)+N \sum_{\mathrm{mj}}^{\infty}\left(\mathrm{t}, \overline{\mathrm{F}}\left(\mathrm{t},\left[R \sum_{i=0}^{\infty} \underline{\mathrm{u}}_{(j m-1) i}(\mathrm{t}, \alpha)+N \sum_{i=0}^{\infty} \overline{\mathrm{u}}_{(j m-1) i}(\mathrm{t}, \alpha)\right]\right)\right) \mathrm{t}, \alpha\right)\right]\right)\right) d t+
\end{gathered}
$$

where $0 \leq \mathrm{t} \leq \mathrm{d}, \mathrm{d} \leq \mathrm{t} \leq \mathrm{c}, \mathrm{c} \leq \mathrm{t} \leq \mathrm{e}, \mathrm{e} \leq \mathrm{t} \leq \mathrm{x}, 0 \leq \alpha \leq 1$

The Modified decomposition method introduces the use of the recurrence relation

$\underline{u}_{0 \mathrm{~m}}(x, \alpha)=\underline{f_{00}}(x, \alpha)$

$$
\begin{aligned}
& \underline{\mathrm{u}_{1 m}}(\mathrm{x}, \alpha)=\underline{f_{11}}(\mathrm{x}, \alpha) \\
& +\sum_{j=1}^{m}\left[\int _ { \mathrm { a } } ^ { \mathrm { d } } \mathrm { k } _ { \mathrm { mj } } \left(\mathrm{x}, \mathrm{t}, \underline{\mathrm{G}_{\mathrm{mj}}}\left(\mathrm{t}, \underline{\mathrm{F}}\left(\mathrm{t},\left[R \sum_{i=0}^{\infty} \underline{\mathrm{u}}_{\mathrm{j}(m-1) 0}(\mathrm{t}, \alpha)+N \sum_{r=0}^{\infty} \underline{\mathrm{u}}_{\mathrm{j}(m-1) 0}(\mathrm{t}, \alpha)\right)\right) \mathrm{dt}\right.\right.\right. \\
& +\int_{\mathrm{d}}^{\mathrm{c}} \mathrm{k}_{12}\left(\mathrm{x}, \mathrm{t}, \overline{\mathrm{G}}_{2}\left(\mathrm{t}, \overline{\mathrm{F}}\left(\mathrm{t},\left[R \sum_{r=0}^{\infty} \overline{\mathrm{u}}_{2(n-1) 0}(\mathrm{t}, \alpha)+N \sum_{r=0}^{\infty} \overline{\mathrm{u}}_{2(n-1) 0}(\mathrm{t}, \alpha)\right]\right)\right) \mathrm{dt}\right. \\
& +\int_{\mathrm{c}}^{\mathrm{e}} \mathrm{k}_{21}\left(\mathrm{x}, \mathrm{t}, \underline{\mathrm{G}}_{1}\left(\mathrm{t}, \overline{\mathrm{F}}\left(\mathrm{t},\left[R \sum_{r=0}^{\infty} \overline{\mathrm{u}}_{1(n-1) r}(\mathrm{t}, \alpha)+N \sum_{r=0}^{\infty} \overline{\mathrm{u}}_{1(n-1) r}(\mathrm{t}, \alpha)\right]\right)\right) \mathrm{dt}\right. \\
& +\int_{\mathrm{e}}^{\mathrm{x}} \mathrm{k}_{22}\left(\mathrm{x}, \mathrm{t}, \overline{\mathrm{G}}_{2}\left(\mathrm{t}, \underline{\mathrm{F}}\left(\mathrm{t},\left[R \sum_{r=0}^{\infty} \underline{\mathrm{u}}_{2(n-1) 0}(\mathrm{t}, \alpha)+N \sum_{r=0}^{\infty} \underline{\underline{u}}_{2(n-1) 0}(\mathrm{t}, \alpha)\right]\right)\right) \mathrm{dt}\right] \\
& \underline{\mathrm{u}_{1 n(r+2)}}(\mathrm{x}, \alpha)=\int_{\mathrm{a}}^{\mathrm{d}} \mathrm{k}_{11}\left(\mathrm{x}, \mathrm{t}, \underline{\mathrm{G}_{1}}\left(\mathrm{t}, \underline{\mathrm{F}}\left(\mathrm{t},\left[R \sum_{r=0}^{\infty} \underline{\underline{u}}_{1(n-1)(r+1)}(\mathrm{t}, \alpha)+N \sum_{r=0}^{\infty} \underline{\underline{u}}_{1(n-1)(r+1)}(\mathrm{t}, \alpha)\right)\right]\right) \mathrm{dt}\right. \\
& +\int_{\mathrm{d}}^{\mathrm{c}} \mathrm{k}_{12}\left(\mathrm{x}, \mathrm{t}, \overline{\mathrm{G}}_{2}\left(\mathrm{t}, \overline{\mathrm{F}}\left(\mathrm{t},\left[R \sum_{r=0}^{\infty} \overline{\mathrm{u}}_{2(n-1)(r+1)}(\mathrm{t}, \alpha)+N \sum_{r=0}^{\infty} \overline{\mathrm{u}}_{2(n-1)(r+1)}(\mathrm{t}, \alpha)\right]\right)\right) \mathrm{dt}\right. \\
& +\int_{\mathrm{c}}^{\mathrm{e}} \mathrm{k}_{21}\left(\mathrm{x}, \mathrm{t}, \underline{\mathrm{G}}_{1}\left(\mathrm{t}, \overline{\mathrm{F}}\left(\mathrm{t},\left[R \sum_{r=0}^{\infty} \overline{\mathrm{u}}_{1(n-1)(r+1)}(\mathrm{t}, \alpha)+N \sum_{r=0}^{\infty} \overline{\mathrm{u}}_{1(n-1)(r+1)}(\mathrm{t}, \alpha)\right]\right)\right) \mathrm{dt}\right. \\
& +\int_{\mathrm{e}}^{\mathrm{x}} \mathrm{k}_{22}\left(\mathrm{x}, \mathrm{t}, \overline{\mathrm{G}}_{2}\left(\mathrm{t}, \underline{\mathrm{F}}\left(\mathrm{t},\left[R \sum_{r=0}^{\infty} \underline{\mathrm{u}}_{2(n-1)(r+1)}(\mathrm{t}, \alpha)+N \sum_{r=0}^{\infty} \underline{\mathrm{u}}_{2(n-1)(r+1)}(\mathrm{t}, \alpha)\right]\right)\right) \mathrm{dt}\right.
\end{aligned}
$$




$$
\begin{aligned}
& \bar{u}_{0 n 0}(x, \alpha)=\overline{f_{00}}(x, \alpha) \\
& \overline{u_{1 n 1}}(\mathrm{x}, \alpha)=\underline{f_{11}}(\mathrm{x}, \alpha)+ \\
& \int_{\mathrm{a}}^{\mathrm{d}} \mathrm{k}_{12}\left(\mathrm{x}, \mathrm{t}, \overline{\mathrm{G}}_{2}\left(\mathrm{t}, \overline{\mathrm{F}}\left(\mathrm{t},\left[R \sum_{r=0}^{\infty} \overline{\mathrm{u}}_{2(n-1) 0}(\mathrm{t}, \alpha)+N \sum_{r=0}^{\infty} \overline{\mathrm{u}}_{2(n-1) 0}(\mathrm{t}, \alpha)\right]\right)\right) \mathrm{dt}+\right. \\
& \int_{d}^{c} \mathrm{k}_{11}\left(\mathrm{x}, \mathrm{t}, \underline{\mathrm{G}_{1}}\left(\mathrm{t}, \underline{\mathrm{F}}\left(\mathrm{t},\left[R \sum_{r=0}^{\infty} \underline{\mathrm{u}}_{1(n-1) 0}(\mathrm{t}, \alpha)+N \sum_{r=0}^{\infty} \underline{\mathrm{u}}_{1(n-1) 0}(\mathrm{t}, \alpha)\right)\right]\right) \mathrm{dt}+\right. \\
& \int_{\mathrm{c}}^{\mathrm{e}} \mathrm{k}_{22}\left(\mathrm{x}, \mathrm{t}, \overline{\mathrm{G}}_{2}\left(\mathrm{t}, \underline{\mathrm{F}}\left(\mathrm{t},\left[R \sum_{r=0}^{\infty} \underline{\mathrm{u}}_{2(n-1) 0}(\mathrm{t}, \alpha)+N \sum_{r=0}^{\infty} \underline{\mathrm{u}}_{2(n-1) 0}(\mathrm{t}, \alpha)\right]\right)\right) \mathrm{dt}+\right. \\
& \int_{\mathrm{e}}^{\mathrm{x}} \mathrm{k}_{21}\left(\mathrm{x}, \mathrm{t}, \underline{\mathrm{G}}_{1}\left(\mathrm{t}, \overline{\mathrm{F}}\left(\mathrm{t},\left[R \sum_{r=0}^{\infty} \overline{\mathrm{u}}_{1(n-1) r}(\mathrm{t}, \alpha)+N \sum_{r=0}^{\infty} \overline{\mathrm{u}}_{1(n-1) r}(\mathrm{t}, \alpha)\right]\right)\right) \mathrm{d}\right. \\
& \bar{u}_{1 n(r+2)}(x, \alpha)=\int_{\mathrm{a}}^{\mathrm{d}} \mathrm{k}_{12}\left(\mathrm{x}, \mathrm{t}, \overline{\mathrm{G}}_{2}\left(\mathrm{t}, \overline{\mathrm{F}}\left(\mathrm{t},\left[R \sum_{r=0}^{\infty} \overline{\mathrm{u}}_{2(n-1)(r+1)}(\mathrm{t}, \alpha)+N \sum_{r=0}^{\infty} \overline{\mathrm{u}}_{2(n-1)(r+1)}(\mathrm{t}, \alpha)\right]\right)\right) \mathrm{dt}+\right. \\
& \int_{d}^{c} \mathrm{k}_{11}\left(\mathrm{x}, \mathrm{t}, \underline{\mathrm{G}_{1}}\left(\mathrm{t}, \underline{\mathrm{F}}\left(\mathrm{t},\left[R \sum_{r=0}^{\infty} \underline{\mathrm{u}}_{1(n-1)(r+1)}(\mathrm{t}, \alpha)+\right.\right.\right.\right. \\
& \left.\left.\left.N \sum_{r=0}^{\infty} \underline{\mathrm{u}}_{1(n-1)(r+1)}(\mathrm{t}, \alpha)\right)\right]\right) d t+\int_{\mathrm{c}}^{\mathrm{e}} \mathrm{k}_{22}\left(\mathrm{x}, \mathrm{t}, \overline{\mathrm{G}}_{2}\left(\mathrm{t}, \underline{\mathrm{F}}\left(\mathrm{t},\left[R \sum_{r=0}^{\infty} \underline{\mathrm{u}}_{2(n-1)(r+1)}(\mathrm{t}, \alpha)+\right.\right.\right.\right. \\
& \left.\left.\left.N \sum_{r=0}^{\infty} \underline{\mathrm{u}}_{2(n-1)(r+1)}(\mathrm{t}, \alpha)\right]\right)\right) \mathrm{dt}+\int_{\mathrm{e}}^{\mathrm{x}} \mathrm{k}_{21}\left(\mathrm{x}, \mathrm{t}, \underline{\mathrm{G}}_{1}\left(\mathrm{t}, \overline{\mathrm{F}}\left(\mathrm{t},\left[R \sum_{r=0}^{\infty} \overline{\mathrm{u}}_{1(n-1)(r+1)}(\mathrm{t}, \alpha)+N \sum_{r=0}^{\infty} \overline{\mathrm{u}}_{1(n-1)(r+1)}(\mathrm{t}, \alpha)\right]\right)\right) \mathrm{dt}\right. \\
& k \geq 0
\end{aligned}
$$

As started before, we many need only two iteration to derive the exact solution. If more than two iteration are needed $\left[N\left(\underline{u}_{j(n-1)(r+1)}(t, \alpha), N\left(\bar{u}_{j(n-1)(r+1)}(t, \alpha)\right)\right]\right.$ should be represented by Adomian polynomial which can easily be generated for all types of non- linearty.

The choice of $\underline{f_{i 0}}(x, \alpha), \bar{f}_{i 0}(x, \alpha)$ cantina the minimal number of terms has a strong influence on facilitating the recurrence relation, and as a consequence, accelerates the convergence of the solution. Also the exact selution must be a term of $\underline{f}_{i}(x, \alpha), \bar{f}_{i}(x, \alpha)$ or to be a part of series of $\underline{f}_{i}(x, \alpha), \bar{f}_{i}(x, \alpha)$.This means that the success of this method depends mainly on the proper choice of $\underline{f_{i 0}}, \bar{f}_{i 0}$ and $\underline{f_{i 1}}, \overline{f_{i 1}}$. We have been unable to establish any criteria to judge what forms of $\underline{f_{i 0}}, \bar{f}_{i 0}$ and $\underline{f_{i 1}}, \overline{f_{i 1}}$ can be used to yield the acceleration demanded. At present $\underline{f_{i 0}}, \bar{f}_{i 0}$ and $\underline{f_{i 1}}, \overline{f_{i 1}}$ are selected by trials. Several illustrative example are used to show the pertinent features of the modified method.

\section{Example 5. Numerical}

Considered the following system

$$
\begin{gathered}
k_{11}\left(x, t, \underline{G}_{1}\left(t, \underline{F}\left(t, \underline{u}_{1 n-1}(t, \alpha)\right)\right)=t^{2}\left(\underline{u}_{1 n-1}(t, \alpha)\right)^{2}\right. \\
k_{12}\left(x, t, \bar{G}_{2}\left(t, \bar{F}\left(t, \bar{u}_{2 n-1}(t, \alpha)\right)\right)=(x-t) t\left(\bar{u}_{2 n-1}(t, \alpha)\right)^{2}\right.
\end{gathered}
$$




$$
\begin{gathered}
k_{21}\left(x, t, \bar{G}_{1}\left(t, \underline{F}\left(t, \underline{u}_{1 n-1}(t, \alpha)\right)\right)=x^{2} t\left(\underline{u}_{1 n-1}(t, \alpha)\right)^{2}\right. \\
k_{22}\left(x, t, \underline{G}_{2}\left(t, \bar{F}\left(t, \bar{u}_{2 n-1}(t, \alpha)\right)\right)=t^{5}\left(\bar{u}_{2 n-1}(t, \alpha)\right)^{2}\right. \\
\begin{array}{l}
\quad+0.0156) \\
f_{1}(x, \alpha)=x \alpha-\left(\frac{x^{9}}{72}+0.00625\right) \alpha^{2}-x\left(0.000217(2-\alpha)^{2}-0.00074 \alpha-0.000217\right)+(2-\alpha)^{2}\left(0.0000977-\frac{x^{4}}{4}\right.
\end{array} \\
\quad \begin{array}{l}
\quad 0.00625) \\
\bar{f}_{1}(x, \alpha)=x(2-\alpha)-\alpha^{2}\left(0.0156+0.000977-\frac{x^{10}}{90}-x\left(0.00049(2-\alpha)^{2}+\alpha^{2}\right)+(2-\alpha)^{2}\left(0.000217-\frac{x^{5}}{5}\right.\right.
\end{array} \\
\underline{f}_{2}(x, \alpha)=x^{2}(2-\alpha)-0.00625 x \alpha^{2}-(2-\alpha)^{2}\left(0.000044+\frac{x^{6}}{4}-0.0156 x^{2}+\frac{x^{10}}{10}-0.000977\right) \\
\bar{f}_{2}(x, \alpha)=x^{2} \alpha-0.0156 x^{2} \alpha^{2}-0.000217(2-\alpha)^{2}-\frac{x^{7}}{5}(2-\alpha)^{2}+x(2-\alpha)^{2} 0.000625-\frac{x^{11}}{11} \alpha^{2}+0.000044 \alpha^{2}
\end{gathered}
$$

The exact solutions for our system are

$$
\begin{aligned}
& \underline{u}_{1}(x, \alpha)=x \alpha \text { and } \bar{u}_{1}(x, \alpha)=x(2-\alpha) \\
& \underline{u}_{2}(x, \alpha)=x^{2}(2-\alpha) \text { and } \bar{u}_{2}(x, \alpha)=x^{2} \alpha
\end{aligned}
$$

$\mathrm{i}=2$ and $\mathrm{m}=2$

$$
\begin{aligned}
& \underline{\mathrm{u}_{1 n}}(\mathrm{x}, \alpha)=\underline{\mathrm{f}}_{1}(\mathrm{x}, \alpha) \\
& +\int_{\mathrm{a}}^{\mathrm{d}} \mathrm{k}_{11}\left(\mathrm{x}, \mathrm{t}, \underline{\mathrm{G}_{1}}\left(\mathrm{t}, \underline{\underline{\mathrm{F}}}\left(\mathrm{t}, \underline{\mathrm{u}}_{1 n-1}(\mathrm{t}, \alpha)\right)\right) \mathrm{dt}+\int_{\mathrm{d}}^{\mathrm{c}} \mathrm{k}_{12}\left(\mathrm{x}, \mathrm{t}, \overline{\mathrm{G}}_{2}\left(\mathrm{t}, \overline{\mathrm{F}}\left(\mathrm{t}, \overline{\mathrm{u}}_{2 n-1}(\mathrm{t}, \alpha)\right)\right) \mathrm{dt}\right.\right. \\
& +\int_{c}^{\mathrm{e}} \mathrm{k}_{21}\left(\mathrm{x}, \mathrm{t}, \underline{G}_{1}\left(\mathrm{t}, \overline{\mathrm{F}}\left(\mathrm{t}, \overline{\mathrm{u}}_{1 n-1}(\mathrm{t}, \alpha)\right)\right) \mathrm{dt}+\int_{\mathrm{e}}^{\mathrm{x}} \mathrm{k}_{22}\left(\mathrm{x}, \mathrm{t}, \overline{\mathrm{G}}_{2}\left(\mathrm{t}, \underline{\mathrm{F}}\left(\mathrm{t}, \underline{\mathrm{u}}_{2 n-1}(\mathrm{t}, \alpha)\right)\right) \mathrm{dt}\right.\right. \\
& \overline{\mathrm{u}_{1 n}}(\mathrm{x}, \alpha)=\overline{\mathrm{f}}_{1}(\mathrm{x}, \alpha)+\int_{\mathrm{a}}^{\mathrm{d}} \mathrm{k}_{11}\left(\mathrm{x}, \mathrm{t}, \overline{\mathrm{G}}_{1}\left(\mathrm{t}, \overline{\mathrm{F}}\left(\mathrm{t}, \overline{\mathrm{u}}_{1 n-1}(\mathrm{t}, \alpha)\right)\right) d t+\int_{\mathrm{d}}^{\mathrm{c}} \mathrm{k}_{12}\left(\mathrm{x}, \mathrm{t}, \mathrm{k}\left(\mathrm{x}, \mathrm{t}, \underline{\mathrm{G}_{2}}\left(\mathrm{t}, \underline{\mathrm{F}}\left(\mathrm{t}, \underline{\mathrm{u}}_{2 n-1}(\mathrm{t}, \alpha)\right)\right) d t+\right.\right.\right. \\
& \int_{\mathrm{c}}^{\mathrm{e}} \mathrm{k}_{21}\left(\mathrm{x}, \mathrm{t}, \overline{\mathrm{G}}_{1}\left(\mathrm{t}, \underline{\mathrm{F}}\left(\mathrm{t}, \underline{\mathrm{u}}_{1 n-1}(\mathrm{t}, \alpha)\right)\right) \mathrm{dt}+\int_{\mathrm{e}}^{\mathrm{x}} \mathrm{k}_{22}\left(\mathrm{x}, \mathrm{t}, \underline{\mathrm{G}}_{2}\left(\mathrm{t}, \overline{\mathrm{F}}\left(\mathrm{t}, \overline{\mathrm{u}}_{2 n-1}(\mathrm{t}, \alpha)\right)\right) \mathrm{dt}\right.\right.
\end{aligned}
$$

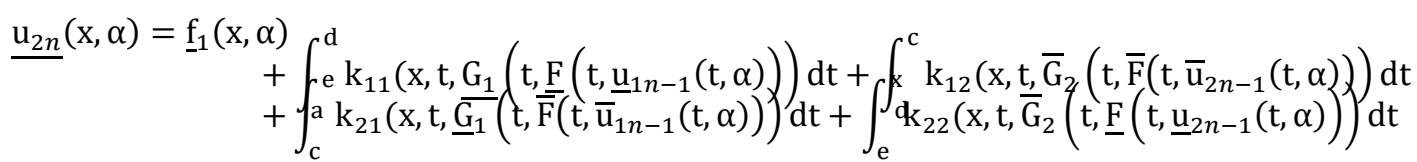

$$
\begin{aligned}
& \overline{\mathrm{u}_{2 n}}(\mathrm{x}, \alpha)=\overline{\mathrm{f}}_{2}(\mathrm{x}, \alpha)+\int_{\mathrm{a}}^{\mathrm{d}} \mathrm{k}_{11}\left(\mathrm{x}, \mathrm{t}, \overline{\mathrm{G}}_{1}\left(\mathrm{t}, \overline{\mathrm{F}}\left(\mathrm{t}, \overline{\mathrm{u}}_{1 n-1}(\mathrm{t}, \alpha)\right)\right) d t+\int_{\mathrm{d}}^{\mathrm{c}} \mathrm{k}_{12}\left(\mathrm{x}, \mathrm{t}, \mathrm{k}\left(\mathrm{x}, \mathrm{t}, \underline{\mathrm{G}_{2}}\left(\mathrm{t}, \underline{\mathrm{F}}\left(\mathrm{t}, \underline{\mathrm{u}}_{2 n-1}(\mathrm{t}, \alpha)\right)\right) d t+\right.\right.\right. \\
& \int_{c}^{\mathrm{e}} \mathrm{k}_{21}\left(\mathrm{x}, \mathrm{t}, \overline{\mathrm{G}}_{1}\left(\mathrm{t}, \underline{\mathrm{F}}\left(\mathrm{t}, \underline{\mathrm{u}}_{1 n-1}(\mathrm{t}, \alpha)\right)\right) \mathrm{dt}+\int_{\mathrm{e}}^{\mathrm{x}} \mathrm{k}_{22}\left(\mathrm{x}, \mathrm{t}, \underline{\mathrm{G}}_{2}\left(\mathrm{t}, \overline{\mathrm{F}}\left(\mathrm{t}, \overline{\mathrm{u}}_{2 n-1}(\mathrm{t}, \alpha)\right)\right) \mathrm{dt}\right.\right.
\end{aligned}
$$

$\mathrm{n}=0,1, \ldots$

Now we applied modified decompositions method to solve our system

$$
\begin{gathered}
f_{1}(x, \alpha)=f_{10}(x, \alpha)+f_{11}(x, \alpha) \\
\bar{f}_{1}(x, \alpha)=\overline{\bar{f}}_{10}(x, \alpha)+\overline{\bar{f}}_{11}(x, \alpha) \\
f_{2}(x, \alpha)=f_{20}(x, \alpha)+f_{21}(x, \alpha) \\
\overline{\bar{f}}_{2}(x, \alpha)=\overline{\bar{f}}_{20}(x, \alpha)+\overline{\bar{f}}_{21}(x, \alpha) \\
\underline{f}_{10}(x, \alpha)=x \alpha
\end{gathered}
$$




$$
\begin{aligned}
& \underline{f_{11}}(x, \alpha)=-\left(\frac{x^{9}}{72}+0.00625\right) \alpha^{2}-x\left(0.000217(2-\alpha)^{2}-0.00074 \alpha-0.000217\right)+(2-\alpha)^{2}\left(0.0000977-\frac{x^{4}}{4}\right. \\
& +0.0156) \\
& \bar{f}_{10}(x, \alpha)=x(2-\alpha) \\
& \bar{f}_{11}(x, \alpha)=-\alpha^{2}\left(0.0156+0.000977-\frac{x^{10}}{90}-x\left(0.00049(2-\alpha)^{2}+\alpha^{2}\right)+(2-\alpha)^{2}\left(0.000217-\frac{x^{5}}{5}+0.00625\right)\right. \\
& \underline{f_{20}}(x, \alpha)=x^{2}(2-\alpha) \\
& \underline{f_{21}}(x, \alpha)=-0.00625 x \alpha^{2}-(2-\alpha)^{2}\left(0.000044+\frac{x^{6}}{4}-0.0156 x^{2}+\frac{x^{10}}{10}-0.000977\right) \\
& \bar{f}_{20}(x, \alpha)=x^{2} \alpha \\
& \bar{f}_{21}(x, \alpha)=-0.0156 x^{2} \alpha^{2}-0.000217(2-\alpha)^{2}-\frac{x^{7}}{5}(2-\alpha)^{2}+x(2-\alpha)^{2} 0.000625-\frac{x^{11}}{11} \alpha^{2}+0.000044 \alpha^{2} \\
& \overline{\mathrm{u}_{10}}(\mathrm{x}, \alpha)=\bar{f}_{10}(x, \alpha)=x(2-\alpha) \\
& \underline{\mathrm{u}_{10}}(\mathrm{x}, \alpha)=\underline{f_{10}}(x, \alpha)=x \alpha \\
& \underline{\mathrm{u}_{20}}(\mathrm{x}, \alpha)=\underline{f_{20}}(x, \alpha)=x^{2}(2-\alpha) \\
& \overline{\mathrm{u}_{20}}(\mathrm{x}, \alpha)=\bar{f}_{20}(x, \alpha)=x^{2} \alpha \\
& \underline{\mathrm{u}_{11}}(\mathrm{x}, \alpha)=\underline{f}_{11}(x, \alpha)+\int_{\mathrm{a}}^{\mathrm{d}} \mathrm{t}^{2}\left(\underline{\mathrm{u}}_{10}(\mathrm{t}, \alpha)\right)^{2} \mathrm{dt}+\int_{\mathrm{d}}^{\mathrm{c}}(\mathrm{x}-\mathrm{t}) \mathrm{t}\left(\overline{\mathrm{u}}_{20}(\mathrm{t}, \alpha)\right)^{2} \mathrm{dt}+\int_{\mathrm{c}}^{\mathrm{e}} x^{2} t\left(\left(\overline{\mathrm{u}}_{10}(\mathrm{t}, \alpha)\right)\right)^{2} \mathrm{dt} \\
& +\int_{\mathrm{e}}^{\mathrm{x}} \mathrm{t}^{5}\left(\left(\underline{\mathrm{u}}_{20}(\mathrm{t}, \alpha)\right)\right)^{2} \mathrm{dt}=0
\end{aligned}
$$

$\overline{\mathrm{u}_{11}}(\mathrm{x}, \alpha)=$

$\bar{f}_{11}(x, \alpha)+\int_{\mathrm{a}}^{\mathrm{d}} \mathrm{t}^{2}\left(\left(\overline{\mathrm{u}}_{10}(\mathrm{t}, \alpha)\right)\right)^{2} d t+\int_{\mathrm{d}}^{\mathrm{c}}(\mathrm{x}-\mathrm{t}) \mathrm{t}\left(\left(\underline{\mathrm{u}}_{20}(\mathrm{t}, \alpha)\right)\right) d t+\int_{\mathrm{c}}^{\mathrm{e}} \mathrm{x}^{2} t\left(\left(\underline{\mathrm{u}}_{10}(\mathrm{t}, \alpha)\right)\right)^{2} \mathrm{dt}+\int_{\mathrm{e}}^{\mathrm{x}} t^{5}\left(\left(\overline{\mathrm{u}}_{20}(\mathrm{t}, \alpha)\right)\right)^{2} \mathrm{dt}$ $=0$

$$
\begin{gathered}
\underline{\mathrm{u}_{21}}(\mathrm{x}, \alpha)=\underline{f}_{21}(x, \alpha)+\int_{\mathrm{a}}^{\mathrm{d}} \mathrm{t}^{2}\left(\left(\underline{\mathrm{u}}_{1 n-1}(\mathrm{t}, \alpha)\right)\right)^{2} \mathrm{dt}+\int_{\mathrm{d}}^{\mathrm{c}}(\mathrm{x}-\mathrm{t}) \mathrm{t}\left(\left(\overline{\mathrm{u}}_{20}(\mathrm{t}, \alpha)\right)\right)^{2} \mathrm{dt}+\int_{\mathrm{c}}^{\mathrm{e}} \mathrm{x}^{2} t\left(\left(\overline{\mathrm{u}}_{10}(\mathrm{t}, \alpha)\right)\right)^{2} \mathrm{dt} \\
+\int_{\mathrm{e}}^{\mathrm{x}} \mathrm{t}^{5}\left(\left(\underline{\mathrm{u}}_{20}(\mathrm{t}, \alpha)\right)\right)^{2} \mathrm{dt}=0 \\
\overline{\mathrm{u}}_{21}(\mathrm{x}, \alpha)= \\
\bar{f}_{21}(x, \alpha)+\int_{\mathrm{a}}^{\mathrm{d}} \mathrm{t}^{2}\left(\left(, \overline{\mathrm{u}}_{10}(\mathrm{t}, \alpha)\right)\right)^{2} d t+\int_{\mathrm{d}}^{\mathrm{c}}(\mathrm{x}-\mathrm{t}) \mathrm{t}\left(\left(\underline{\mathrm{u}}_{20}(\mathrm{t}, \alpha)\right)\right)^{2} d t+\int_{\mathrm{c}}^{\mathrm{e}} \mathrm{x}^{2} t\left(\left(\underline{\mathrm{u}}_{10}(\mathrm{t}, \alpha)\right)\right)^{2} \mathrm{dt}+\int_{\mathrm{e}}^{\mathrm{x}} \mathrm{t}^{5}\left(\left(\overline{\mathrm{u}}_{20}(\mathrm{t}, \alpha)\right)\right)^{2} \mathrm{dt} \\
=0
\end{gathered}
$$

where $a=0, d=0.25, c=0.5, e=0.75$ 
Table 1. compered between the exact solution and MDM for $\underline{\mathrm{u}_{11}}$

\begin{tabular}{|l|l|l|l|l|l|l|l|l|l|l|l|l|l|l|l|l|l|l|}
\hline X & \multicolumn{10}{|c|}{$\mathrm{u}_{11}$} \\
\hline
\end{tabular}

Table 2. compered between the exact solution and MDM for $\bar{u}_{11}$

\begin{tabular}{|l|l|l|l|l|l|l|l|l|l|l|l|l|l|l|l|l|l|l|}
\hline X & \multicolumn{10}{|c|}{$\bar{n}_{11}$} \\
\multicolumn{10}{|c|}{ MDM } \\
\hline$\alpha$ & 0.1 & 0.3 & 0.5 & 0.7 & 0.9 & 1.0 & 0.1 & 0.3 & 0.5 & 0.7 & 0.9 & 1.0 & 0.1 & 0.3 & 0. & 0.7 & 0.9 & 1.0 \\
\hline 0 & 0.0 & 0.0 & 0.0 & 0.0 & 0.0 & 0.0 & 0.0 & 0.0 & 0.0 & 0.0 & 0.0 & 0.0 & 000 & 00 & 00 & 00 & 00 & 00 \\
& 0 & 0 & 0 & 0 & 0 & 0 & 0 & 0 & 0 & 0 & 0 & 0 & 0 & 0 & 0 & 0 & 0 & 0 \\
\hline 0. & 0.3 & 0.3 & 0.3 & 0.2 & 0.2 & 0.2 & 0.3 & 0.3 & 0.3 & 0.2 & 0.2 & 0.2 & 000 & 00 & 00 & 00 & 00 & 00 \\
2 & 8 & 4 & 0 & 6 & 2 & 0 & 8 & 4 & 0 & 6 & 2 & 0 & 0 & 0 & 0 & 0 & 0 & 0 \\
\hline 0. & 0.7 & 0.6 & 0.6 & 0.5 & 0.4 & 0.4 & 0.7 & 0.6 & 0.6 & 0.5 & 0.4 & 0.4 & 000 & 00 & 00 & 00 & 00 & 00 \\
4 & 6 & 8 & 0 & 2 & 4 & 0 & 6 & 8 & 0 & 2 & 4 & 0 & 0 & 0 & 0 & 0 & 0 & 0 \\
\hline 0. & 1.1 & 1.0 & 0.9 & 0.7 & 0.6 & 0.6 & 1.1 & 1.0 & 0.9 & 0.7 & 0.6 & 0.6 & 000 & 00 & 00 & 00 & 00 & 00 \\
6 & 4 & 2 & 0 & 8 & 6 & 0 & 4 & 2 & 0 & 8 & 6 & 0 & 0 & 0 & 0 & 0 & 0 & 0 \\
\hline 0. & 1.5 & 1.3 & 1.2 & 1.0 & 0.8 & 0.8 & 1.5 & 1.3 & 1.2 & 1.0 & 0.8 & 0.8 & 000 & 00 & 00 & 00 & 00 & 00 \\
8 & 2 & 6 & 0 & 4 & 8 & 0 & 2 & 6 & 0 & 4 & 8 & 0 & 0 & 0 & 0 & 0 & 0 & 0 \\
\hline 1. & 1.9 & 1.7 & 1.5 & 1.3 & 1.1 & 1.0 & 1.9 & 1.7 & 1.5 & 1.3 & 1.1 & 1.0 & 000 & 00 & 00 & 00 & 00 & 00 \\
0 & 0 & 0 & 0 & 0 & 1 & 0 & 0 & 0 & 0 & 0 & 1 & 0 & 0 & 0 & 0 & 0 & 0 & 0 \\
\hline
\end{tabular}


Table 3. compered between the exact solution and MDM for $\underline{\mathrm{u}_{12}}$

\begin{tabular}{|c|c|c|c|c|c|c|c|c|c|c|c|c|c|c|c|c|c|c|}
\hline $\mathrm{x}$ & \multicolumn{6}{|c|}{$\mathrm{u}_{12}$} & \multicolumn{6}{|c|}{ MDM } & \multicolumn{6}{|c|}{ Absolute error } \\
\hline$\alpha$ & 0.1 & 0.3 & $\begin{array}{l}0 . \\
5\end{array}$ & 0.7 & 0.9 & 1.0 & 0.1 & 0.3 & $\begin{array}{l}0 . \\
5\end{array}$ & 0.7 & 0.9 & 1.0 & 0.1 & 0.3 & 0. & 0.7 & 0.9 & 1.0 \\
\hline 0 & 00 & 00 & 00 & 00 & 00 & 00 & 00 & 00 & 00 & 00 & 00 & 00 & $\begin{array}{l}000 \\
0\end{array}$ & $\begin{array}{l}00 \\
0\end{array}$ & $\begin{array}{l}00 \\
0\end{array}$ & $\begin{array}{l}00 \\
0\end{array}$ & $\begin{array}{l}00 \\
0\end{array}$ & $\begin{array}{l}00 \\
0\end{array}$ \\
\hline $\begin{array}{l}0 . \\
2\end{array}$ & $\begin{array}{l}.07 \\
6\end{array}$ & .068 & $\begin{array}{l}.0 \\
6\end{array}$ & $\begin{array}{l}.05 \\
2\end{array}$ & $\begin{array}{l}.04 \\
4\end{array}$ & $\begin{array}{l}0.0 \\
4\end{array}$ & $\begin{array}{l}.07 \\
6\end{array}$ & .068 & $\begin{array}{l}.0 \\
6\end{array}$ & $\begin{array}{l}.05 \\
2\end{array}$ & $\begin{array}{l}.04 \\
4\end{array}$ & $\begin{array}{l}0.0 \\
4\end{array}$ & $\begin{array}{l}000 \\
0\end{array}$ & $\begin{array}{l}00 \\
0\end{array}$ & $\begin{array}{l}00 \\
0\end{array}$ & $\begin{array}{l}00 \\
0\end{array}$ & $\begin{array}{l}00 \\
0\end{array}$ & $\begin{array}{l}00 \\
0\end{array}$ \\
\hline $\begin{array}{l}0 . \\
4\end{array}$ & $\begin{array}{l}.30 \\
4\end{array}$ & .272 & $\begin{array}{l}.2 \\
4\end{array}$ & $\begin{array}{l}.20 \\
8\end{array}$ & $\begin{array}{l}.17 \\
6\end{array}$ & $\begin{array}{l}0.1 \\
6\end{array}$ & $\begin{array}{l}.30 \\
4\end{array}$ & .272 & $\begin{array}{l}.2 \\
4\end{array}$ & $\begin{array}{l}.20 \\
8\end{array}$ & $\begin{array}{l}.17 \\
6\end{array}$ & $\begin{array}{l}0.1 \\
6\end{array}$ & $\begin{array}{l}000 \\
0\end{array}$ & $\begin{array}{l}00 \\
0\end{array}$ & $\begin{array}{l}00 \\
0\end{array}$ & $\begin{array}{l}00 \\
0\end{array}$ & $\begin{array}{l}00 \\
0\end{array}$ & $\begin{array}{l}00 \\
0\end{array}$ \\
\hline $\begin{array}{l}0 . \\
6\end{array}$ & $\begin{array}{l}.68 \\
4\end{array}$ & .612 & $\begin{array}{l}.5 \\
4\end{array}$ & $\begin{array}{l}.61 \\
2\end{array}$ & $\begin{array}{l}.39 \\
6\end{array}$ & $\begin{array}{l}0.3 \\
6\end{array}$ & $\begin{array}{l}.68 \\
4\end{array}$ & .612 & $\begin{array}{l}.5 \\
4\end{array}$ & $\begin{array}{l}.61 \\
2\end{array}$ & $\begin{array}{l}.39 \\
6\end{array}$ & $\begin{array}{l}0.3 \\
6\end{array}$ & $\begin{array}{l}000 \\
0\end{array}$ & $\begin{array}{l}00 \\
0\end{array}$ & $\begin{array}{l}00 \\
0\end{array}$ & $\begin{array}{l}00 \\
0\end{array}$ & $\begin{array}{l}00 \\
0\end{array}$ & $\begin{array}{l}00 \\
0\end{array}$ \\
\hline $\begin{array}{l}0 . \\
8\end{array}$ & $\begin{array}{l}1.2 \\
2\end{array}$ & $\begin{array}{l}1.08 \\
8\end{array}$ & $\begin{array}{l}.9 \\
6\end{array}$ & $\begin{array}{l}.83 \\
2\end{array}$ & $\begin{array}{l}.70 \\
4\end{array}$ & $\begin{array}{l}0.6 \\
4\end{array}$ & $\begin{array}{l}1.2 \\
2\end{array}$ & $\begin{array}{l}1.08 \\
8\end{array}$ & $\begin{array}{l}.9 \\
6\end{array}$ & $\begin{array}{l}.83 \\
2\end{array}$ & $\begin{array}{l}.70 \\
4\end{array}$ & $\begin{array}{l}0.6 \\
4\end{array}$ & $\begin{array}{l}000 \\
0\end{array}$ & $\begin{array}{l}00 \\
0\end{array}$ & $\begin{array}{l}00 \\
0\end{array}$ & $\begin{array}{l}00 \\
0\end{array}$ & $\begin{array}{l}00 \\
0\end{array}$ & $\begin{array}{l}00 \\
0\end{array}$ \\
\hline $\begin{array}{l}1 . \\
0\end{array}$ & 1.9 & 1.7 & $\begin{array}{l}1 . \\
5\end{array}$ & 1.3 & 1.1 & 1.0 & 1.9 & 1.7 & $\begin{array}{l}1 . \\
5\end{array}$ & 1.3 & 1.1 & 1.0 & $\begin{array}{l}000 \\
0\end{array}$ & $\begin{array}{l}00 \\
0\end{array}$ & $\begin{array}{l}00 \\
0\end{array}$ & $\begin{array}{l}00 \\
0\end{array}$ & $\begin{array}{l}00 \\
0\end{array}$ & $\begin{array}{l}00 \\
0\end{array}$ \\
\hline
\end{tabular}

Table 4. compered between the exact solution and MDM for $\bar{u}_{22}$

\begin{tabular}{|c|c|c|c|c|c|c|c|c|c|c|c|c|c|c|c|c|c|c|}
\hline$X$ & \multicolumn{6}{|c|}{$\bar{u}_{22}$} & \multicolumn{6}{|c|}{ MDM } & \multicolumn{6}{|c|}{ Absolute error } \\
\hline$\alpha$ & 0.1 & 0.3 & $\begin{array}{l}0 . \\
5\end{array}$ & 0.7 & 0.9 & 1.0 & 0.1 & 0.3 & $\begin{array}{l}0 . \\
5\end{array}$ & 0.7 & 0.9 & 1.0 & 0.1 & 0.3 & 0. & 0.7 & 0.9 & 1.0 \\
\hline 0 & 000 & 00 & $\begin{array}{l}.0 \\
0\end{array}$ & 000 & 000 & 000 & 000 & 00 & $\begin{array}{l}.0 \\
0\end{array}$ & 000 & 000 & 000 & $\begin{array}{l}000 \\
0\end{array}$ & $\begin{array}{l}00 \\
0\end{array}$ & $\begin{array}{l}00 \\
0\end{array}$ & $\begin{array}{l}00 \\
0\end{array}$ & $\begin{array}{l}00 \\
0\end{array}$ & $\begin{array}{l}00 \\
0\end{array}$ \\
\hline $\begin{array}{l}0 . \\
2\end{array}$ & $\begin{array}{l}.00 \\
4\end{array}$ & $\begin{array}{l}.01 \\
2\end{array}$ & $\begin{array}{l}.0 \\
2\end{array}$ & $\begin{array}{l}.02 \\
8\end{array}$ & $\begin{array}{l}.03 \\
6\end{array}$ & $\begin{array}{l}0.0 \\
4\end{array}$ & $\begin{array}{l}.00 \\
4\end{array}$ & $\begin{array}{l}.01 \\
2\end{array}$ & $\begin{array}{l}.0 \\
2\end{array}$ & $\begin{array}{l}.02 \\
8\end{array}$ & $\begin{array}{l}.03 \\
6\end{array}$ & $\begin{array}{l}0.0 \\
4\end{array}$ & $\begin{array}{l}000 \\
0\end{array}$ & $\begin{array}{l}00 \\
0\end{array}$ & $\begin{array}{l}00 \\
0\end{array}$ & $\begin{array}{l}00 \\
0\end{array}$ & $\begin{array}{l}00 \\
0\end{array}$ & $\begin{array}{l}00 \\
0\end{array}$ \\
\hline $\begin{array}{l}0 . \\
4\end{array}$ & $\begin{array}{l}.01 \\
6\end{array}$ & $\begin{array}{l}.04 \\
8\end{array}$ & $\begin{array}{l}.0 \\
8\end{array}$ & $\begin{array}{l}.11 \\
2\end{array}$ & $\begin{array}{l}.14 \\
4\end{array}$ & $\begin{array}{l}0.1 \\
6\end{array}$ & $\begin{array}{l}.01 \\
6\end{array}$ & $\begin{array}{l}.04 \\
8\end{array}$ & $\begin{array}{l}.0 \\
8\end{array}$ & $\begin{array}{l}.11 \\
2\end{array}$ & $\begin{array}{l}.14 \\
4\end{array}$ & $\begin{array}{l}0.1 \\
6\end{array}$ & $\begin{array}{l}000 \\
0\end{array}$ & $\begin{array}{l}00 \\
0\end{array}$ & $\begin{array}{l}00 \\
0\end{array}$ & $\begin{array}{l}00 \\
0\end{array}$ & $\begin{array}{l}00 \\
0\end{array}$ & $\begin{array}{l}00 \\
0\end{array}$ \\
\hline $\begin{array}{l}0 . \\
6\end{array}$ & $\begin{array}{l}.03 \\
6\end{array}$ & $\begin{array}{l}.10 \\
8\end{array}$ & $\begin{array}{l}.1 \\
8\end{array}$ & $\begin{array}{l}.25 \\
2\end{array}$ & $\begin{array}{l}.32 \\
4\end{array}$ & $\begin{array}{l}0.3 \\
6\end{array}$ & $\begin{array}{l}.03 \\
6\end{array}$ & $\begin{array}{l}.10 \\
8\end{array}$ & $\begin{array}{l}.1 \\
8\end{array}$ & $\begin{array}{l}.25 \\
2\end{array}$ & $\begin{array}{l}.32 \\
4\end{array}$ & $\begin{array}{l}0.3 \\
6\end{array}$ & $\begin{array}{l}000 \\
0\end{array}$ & $\begin{array}{l}00 \\
0\end{array}$ & $\begin{array}{l}00 \\
0\end{array}$ & $\begin{array}{l}00 \\
0\end{array}$ & $\begin{array}{l}00 \\
0\end{array}$ & $\begin{array}{l}00 \\
0\end{array}$ \\
\hline $\begin{array}{l}0 . \\
8\end{array}$ & $\begin{array}{l}.06 \\
4\end{array}$ & $\begin{array}{l}.19 \\
2\end{array}$ & $\begin{array}{l}.3 \\
2\end{array}$ & $\begin{array}{l}.44 \\
8\end{array}$ & $\begin{array}{l}.57 \\
6\end{array}$ & $\begin{array}{l}0.6 \\
4\end{array}$ & $\begin{array}{l}.06 \\
4\end{array}$ & $\begin{array}{l}.19 \\
2\end{array}$ & $\begin{array}{l}.3 \\
2\end{array}$ & $\begin{array}{l}.44 \\
8\end{array}$ & $\begin{array}{l}.57 \\
6\end{array}$ & $\begin{array}{l}0.6 \\
4\end{array}$ & $\begin{array}{l}000 \\
0\end{array}$ & $\begin{array}{l}00 \\
0\end{array}$ & $\begin{array}{l}00 \\
0\end{array}$ & $\begin{array}{l}00 \\
0\end{array}$ & $\begin{array}{l}00 \\
0\end{array}$ & $\begin{array}{l}00 \\
0\end{array}$ \\
\hline $\begin{array}{l}1 . \\
0\end{array}$ & 0.1 & 0.3 & $\begin{array}{l}0 . \\
5\end{array}$ & 0.7 & 0.9 & 1.0 & 0.1 & 0.3 & $\begin{array}{l}0 . \\
5\end{array}$ & 0.7 & 0.9 & 1.0 & $\begin{array}{l}000 \\
0\end{array}$ & $\begin{array}{l}00 \\
0\end{array}$ & $\begin{array}{l}00 \\
0\end{array}$ & $\begin{array}{l}00 \\
0\end{array}$ & $\begin{array}{l}00 \\
0\end{array}$ & $\begin{array}{l}00 \\
0\end{array}$ \\
\hline
\end{tabular}

\section{Conclusion}

This paper we are using technique for modified decomposition method to solve system of fuzzy nonlinear integral equation, the method above is implemented in a straight forward manner and proved by using only two iteration to get the exact solution . this technique is so fast and easy to get the converge for solve any equation and system for any value of $\alpha$. The modified admian method there is one important note the exact solution must be a part of the nonhomogeneous function or series of nonhomogeneous 


\section{References}

Abbasbandy, S., \& Allahviranloo, T. (2004). Numerical solution of fuzzy di_erential equation by Runge-Kutta method. Nonlinear Studies, 11, 117-129.

Abbasbandy, S., Nieto, J. J., \& Alavi, M. (2005). Tuning of reachable set in one dimensional fuzzy di_erential inclusions. Chaos, Solitons and Fractals, 26, 1337-1341.

Adomian, G. (1988) A review of the decomposition method in applied mathematics. J. Math. Anal. Appl., 135, $501-544$. http://dx.doi.org/10.1016/0022-247X(88)90170-9.

Allahviranloo, T. (2005) The Adomian decomposition method for fuzzy system of linear equations. Applied mathematics and computation, 163, 553-563. http://dx.doi.org/10.1016/j.amc.2004.02.020.

Allahviranloo, T., Khezerloo, M., Ghanbari, M., \& Khezerloo, S. (2010). The homotopy perturbationmethod for fuzzy Volterra integral equations. International Journal of Cognitive Informatics and Natural Intelligence, 8, 31-37.

Babolian, E., Biazar, J., \& Vahidi, A.R. (2004). Solution of a system of nonlinear equations by Adomian decomposition method. Appl. Math. Comput. 150, 847-854. http://dx.doi.org/10.1016/S0096-3003(03)00313-8

Babolian, E., Sadeghi Goghary, H., \& Abbasbandy, S. (2006). Numerical solution of linear Fredholm fuzzy integral equations of the second kind by Adomian method. Applied Mathematics and Computation, 182, 791-796.

Bildik, N. M. Inc, (2007) Modified decomposition method for nonlinear Volterra-Fredholm integral equations. Chaos, Solitons and Fractals,33, 308-313. http://dx.doi.org/10.1016/j.chaos.2005.12.058.

Caldas, M., \& Jafari, S. (2005). h-Compact fuzzy topological spaces, Chaos, Solitons and Fractals, 25, $229-232$.

Dubois, D., \& Prade, H. (1978). Operations on fuzzy numbers. Int. J. systems Science, 9. 613-626. http://dx.doi.org/10.1080/00207727808941724.

Dubois, D., \& Prade, H. (1982) Towards fuzzy differential calculus. Fuzzy Sets and Systems, 8, 1-7. http://dx.doi.org/10.1016/0165-0114(82)90025-2.

Dubois, D., Prade. (1986). H. Theory and application. fuzzy sets and systems. Academic Press,

Feng, G., \& Chen, G. (2005). Adaptive control of discrete-time chaotic systems: a fuzzy control approach. Chaos, Solitonsand Fractals, 23, 459-467.

Goetschel, R., \& Voxman, W. (1980). Elementary calculus. Fuzzy Sets and Systems, 18, 31-43. http://dx.doi.org/10.1016/0165-0114(86)90026-6.

Jahantigh, M., Allahviranloo,T., \& Otadi, M. (2008) Numerical solution of fuzzy integral equation. Applied Mathematical Sciences, 2, 33-46.

Jiang, W., Guo-Dong, Q., \& Bin. (2005). D. H1 variable universe adaptive fuzzy control for chaotic system. Chaos, Solitonsand Fractals, 24, 1075-1086.

Kaleva, O. (1987) Fuzzy differential equations. Fuzzy Sets and Systems. 24, 301-317. http://dx.doi.org/10.1016/0165-0114(87)90029-7.

Naschie, M. S. El. (2004). A review of E-in_nity theory and the mass spectrum of high energy particle physics. Chaos, Solitons and Fractals, 19, 209-236.

Park, J. H. (2004). Intuitionistic fuzzy metric spaces. Chaos, Solitons and Fractals, 22, 1039-1046.

Tanaka, Y., Mizuno, Y., \& Kado, T. (2005). Chaotic dynamics in the Friedman equation. Chaos, Solitons and Fractals, 24, 407-422.

\section{Copyrights}

Copyright for this article is retained by the author(s), with first publication rights granted to the journal.

This is an open-access article distributed under the terms and conditions of the Creative Commons Attribution license (http://creativecommons.org/licenses/by/4.0/). 